\title{
Hydrogen molecules trapped by multivacancies in silicon
}

\author{
K. Ishioka and M. Kitajima \\ National Research Institute for Metals, Sengen, Tsukuba, Japan
}

S. Tateishi, K. Nakanoya, N. Fukata, ${ }^{*}$ T. Mori, and K. Murakami

Institute of Material Science, University of Tsukuba, Tennoudai, Tsukuba, Japan

S. Hishita

National Institute for Research in Inorganic Materials, Namiki, Tsukuba, Japan

(Received 15 July 1999)

\begin{abstract}
We report an observation of a Raman line of $\mathrm{H}_{2}$ in silicon after $\mathrm{Si}^{+}$-ion implantation followed by a hydrogen atom treatment. The vibrational frequency of the $\mathrm{H}_{2}$ is $3822 \mathrm{~cm}^{-1}$ between the two different vibrational frequencies, 4158 and $3601 \mathrm{~cm}^{-1}$, of $\mathrm{H}_{2}$ observed so far in silicon. The assignment is confirmed by the observation of isotope shifts to $2770 \mathrm{~cm}^{-1}$ for $\mathrm{D}_{2}$ and to $3353 \mathrm{~cm}^{-1}$ for $\mathrm{HD}$. The ion-fluence dependence of the Raman intensity of the $\mathrm{H}_{2}$ at $3822 \mathrm{~cm}^{-1}$ correlates with the total intensity of peaks in the Si-H stretching region that are attributed to $\mathrm{H}$-terminated dangling bonds in multivacancies and/or interstitial-H complexes. We propose that the hydrogen molecule corresponding to the $3822 \mathrm{~cm}^{-1}$ vibrational line is trapped in or adjacent to H-terminated multivacancies. [S0163-1829(99)12739-5]
\end{abstract}

Hydrogen molecules in crystalline semiconductors have attracted much experimental and theoretical attention since their observation by means of Raman spectroscopy in 1996. ${ }^{1,2}$ Murakami and co-workers have revealed the existence of $\mathrm{H}_{2}$ in the float zone (FZ) $n$ - and $p$-type crystalline silicon treated with atomic hydrogen. ${ }^{1,3}$ The $S_{0}(1)$ rotational and $Q_{1}$ vibrational Raman lines of $\mathrm{H}_{2}$ in crystalline silicon appear at 587 and $4158 \mathrm{~cm}^{-1}$, respectively [we label this hydrogen molecule as $\left.\mathrm{H}_{2}(\mathrm{I})\right]$. The observed Raman shifts are very close to those of gaseous hydrogen, whereas their widths are much broader. The small peak shift suggests little interaction with the surrounding host lattice. It has been discussed that these Raman lines are attributable to hydrogen molecules trapped in platelets, or planar defects aligned along the $\{111\}$ plane at tens of nanometers scale. ${ }^{4}$

Pritchard et al. have reported three infrared- (IR-) absorption lines due to $\mathrm{H}-\mathrm{H}$ pair defects in Czochralski (CZ) silicon after exposure to hydrogen gas at temperatures between 1100 and $1300{ }^{\circ} \mathrm{C} .^{5}$ From the isotope shifts the absorption lines have been attributed to hydrogen molecules. Two of the absorption lines, at 3788.9 and $3730.8 \mathrm{~cm}^{-1}$, correlate in their intensities with the vibrational line of interstitial oxygen atoms $\left(\mathrm{O}_{i}\right)$ and have been assigned as $\mathrm{H}_{2}$ adjacent to $\mathrm{O}_{i}$ atoms. The other absorption line, at $3618.3 \mathrm{~cm}^{-1}$ at $10 \mathrm{~K}$, is not correlated with the absorption due to $\mathrm{O}_{i}$ and is observed also in FZ silicon. The downshift in the vibrational frequencies with respect to gaseous hydrogen implies the $\mathrm{H}-\mathrm{H}$ bond is weakened because of the interaction with the surroundings. Very recently a Raman line has also been observed at 3601 $\mathrm{cm}^{-1}$ at room temperature [we label it as $\mathrm{H}_{2}(\mathrm{II})$ ] in a $\mathrm{FZ}$ $n$-type silicon exposed to a hydrogen plasma at $150{ }^{\circ} \mathrm{C}$. ${ }^{6}$ The Raman line shifts to $3618 \mathrm{~cm}^{-1}$ when measured at $10 \mathrm{~K}$, showing a good agreement with the IR result. The width of the $\mathrm{H}_{2}$ (II) Raman line is approximately $6 \mathrm{~cm}^{-1}$, significantly smaller than that of $\mathrm{H}_{2}(\mathrm{I})$ but still greater than that of gaseous hydrogen.

Several theoretical calculations showed that $\mathrm{H}_{2}$ is stable at the tetrahedral (Td) site of the silicon lattice. ${ }^{7-9}$ They pre- dicted a charge redistribution between $\mathrm{H}_{2}$ and the surrounding Si lattice, which results in the screening of the intramolecular proton-electron attraction and the weakening of the $\mathrm{H}_{2}$ bond. On the bases of the computational results the Raman line of $\mathrm{H}_{2}$ (II) has been attributed to hydrogen molecules at Td sites of silicon. ${ }^{6}$

The difference in the vibrational frequencies of the two Raman lines, $\mathrm{H}_{2}$ (I) and $\mathrm{H}_{2}(\mathrm{II})$, is accounted for in terms of different interactions with the surroundings in different trapping environments; platelets and Td sites are the two extremes in terms of the size. It is then reasonable to expect a third vibrational frequency for $\mathrm{H}_{2}$, if any, that exists stably in a trap with a medium size. Such medium-sized traps will be created by ion implantation into crystalline silicon, ${ }^{10,11}$ and the $\mathrm{Si}$ dangling bonds will be terminated with $\mathrm{H}$ atoms by hydrogenation. In the present study we succeeded in observing $\mathrm{H}_{2}$ trapped in multivacancies in silicon using Ramanscattering spectroscopy. Multivacancies were introduced into silicon in a controlled manner by means of $\mathrm{Si}^{+}$-ion implantation. The vibrational frequency of the $\mathrm{H}_{2}$ trapped by multivacancies was found to be between those of $\mathrm{H}_{2}(\mathrm{I})$ and $\mathrm{H}_{2}$ (II).

FZ p-type $\mathrm{Si}(100)$ wafers were used as crystalline silicon samples. In order to create point-defect complexes, i.e., multivacancies and interstitial complexes, we implanted 200$\mathrm{keV} \mathrm{Si}{ }^{+}$ions in the crystalline silicon at fluences ranging from $1 \times 10^{13}$ to $5 \times 10^{15} \mathrm{Si} / \mathrm{cm}^{2}$ at room temperature. It is well known that vacancies formed as a primary product under ion implantation migrate quickly at room temperature and end up with multivacancies and impurity-vacancy complexes. ${ }^{10,11}$ Much less is known concerning the final state of interstitials; most likely they form interstitial complexes, or otherwise diffuse to the surface. The macroscopic degree of disorder of the ion-implanted silicon was estimated in terms of phonon correlation length by applying the spatial correlation model, ${ }^{12}$ to the Raman line shape of the optical phonon. Phonon correlation length, which corresponds to the dimension of phonon delocalization, was obtained to be 500, 


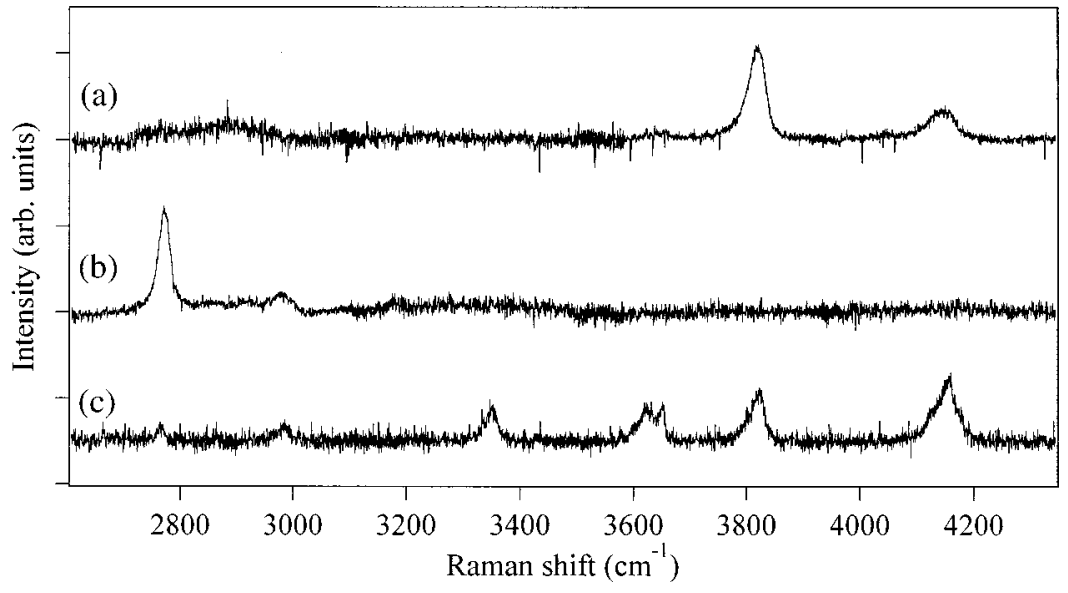

FIG. 1. Raman spectra of silicon after implantation with $200-\mathrm{keV} \mathrm{Si}^{+}$ions at a fluence of $2 \times 10^{14} / \mathrm{cm}^{2}$ followed by a treatment with (a) $\mathrm{H}$ atoms, (b) D atoms, and (c) $\mathrm{H}+\mathrm{D}$ atoms at $250{ }^{\circ} \mathrm{C}$ for $3 \mathrm{~h}$. The smaller relative intensity of $\mathrm{H}_{2}(\mathrm{III})$ to $\mathrm{H}_{2}(\mathrm{I})$ in (c) compared with (a) is attributed to a higher flux of ion implantation that may lead to annealing of defects.

$34.9,17.4,16.5$, and $15.8 \mathrm{~nm}$ for silicon after the implantation of $1 \times 10^{13}, 1 \times 10^{14}, 5 \times 10^{14}, \quad 1 \times 10^{15}$, and $2 \times 10^{15} \mathrm{Si}^{+} / \mathrm{cm}^{2}$, respectively. ${ }^{13}$ The Raman spectrum of the silicon after implantation with a dose of $5 \times 10^{15} \mathrm{Si}^{+} / \mathrm{cm}^{2}$ showed the formation of a continuous amorphous layer.

The implanted samples were treated with atomic hydrogen at $250{ }^{\circ} \mathrm{C}$ for $3 \mathrm{~h}$ in a remote downstream of hydrogen plasma. The samples were placed at a distance $60-\mathrm{cm}$ apart from the plasma to suppress damage from the activated species in the plasma. Details of the hydrogen-atom treatment are described elsewhere. ${ }^{14}$ Atom treatments were also performed replacing hydrogen gas with deuterium gas or with 0.5:0.5 hydrogen-deuterium mixture to check the isotope shift. All the Raman-scattering measurements were performed at room temperature using a 514.5-nm light as an excitation source. The wave-number resolution for the local vibrational mode was $3 \mathrm{~cm}^{-1}$. The optical penetration depth for the 514.5-nm light is approximately $500 \mathrm{~nm}$ for crystal silicon and covers the average projectile range of the 200$\mathrm{keV} \mathrm{Si}$ ions, $250 \mathrm{~nm}$ from the surface.

Figure 1 shows the typical Raman spectra of hydrogen molecules in silicon implanted with $2 \times 10^{14} \mathrm{Si}^{+} / \mathrm{cm}^{2}$ followed by the hydrogen-atom treatment. Two Raman lines were observed at 4158 and $3822 \mathrm{~cm}^{-1}$. The $4158-\mathrm{cm}^{-1}$ line is the $Q_{1}$ vibrational line of $\mathrm{H}_{2}(\mathrm{I})$ that had been observed in unimplanted silicon after hydrogenation. ${ }^{1-3}$ We believe the $3822-\mathrm{cm}^{-1}$ line is observed for the first time in the present study, and appears only for the silicon samples implanted with fluences between $1 \times 10^{13}$ and $5 \times 10^{14} \mathrm{Si}^{+} / \mathrm{cm}^{2}$. No Raman signals due to hydrogenation are observed at around $3600 \mathrm{~cm}^{-1}$ for any implanted samples studied in the present study.

To investigate the origin of the $3822-\mathrm{cm}^{-1}$ peak we performed a deuterium (D) atom treatment, as well as a treatment using a hydrogen-deuterium mixture $(\mathrm{H}+\mathrm{D}$ atom treatment), at $250{ }^{\circ} \mathrm{C}$ for $3 \mathrm{~h}$. The Raman spectra of silicon after implantation with $2 \times 10^{14} \mathrm{Si}^{+} / \mathrm{cm}^{2}$ followed by an isotopic hydrogen atom treatment are compared in Fig. 1. The $3822-\mathrm{cm}^{-1}$ line shows an isotope shift down to $2770 \mathrm{~cm}^{-1}$ for a $\mathrm{D}$ atom treatment. The ratio between the vibrational frequencies is 1.38, in good agreement with that between gaseous $\mathrm{H}_{2}$ and $\mathrm{D}_{2}, 1.39$. After the $\mathrm{H}+\mathrm{D}$ atom treatment a peak was observed at $3353 \mathrm{~cm}^{-1}$ in addition to those at 3822 and $2770 \mathrm{~cm}^{-1}$, which is apparently attributed to the HD molecule. The isotope shifts confirm that the $3822-\mathrm{cm}^{-1}$ line arises from $\mathrm{H}_{2}$ in silicon [we label this hydrogen molecule as $\left.\mathrm{H}_{2}(\mathrm{III})\right]$, and not from any $\mathrm{XH}_{2}$ complexes (e.g., $\mathrm{H}_{2} \mathrm{O}$ ).

Figure 2 summarizes the integrated Raman intensities of the two vibrational lines of $\mathrm{H}_{2}$ as a function of the implantation fluence. The intensities are normalized by that of the optical phonon of silicon. The intensity of $\mathrm{H}_{2}(\mathrm{I})$ decreases monotonically with increasing implantation fluence, whereas that of $\mathrm{H}_{2}$ (III) has a maximum at $2 \times 10^{14} \mathrm{Si}^{+} / \mathrm{cm}^{2}$. The ionfluence dependence indicates that $\mathrm{H}_{2}(\mathrm{III})$ is not related to defects created during hydrogenation (e.g., platelets), but to those created by ion implantation. The downshift in the vibrational frequency, ca. $340 \mathrm{~cm}^{-1}$ from that of gaseous hydrogen, suggests that the interaction of $\mathrm{H}_{2}$ (III) with a silicon lattice is stronger than $\mathrm{H}_{2}(\mathrm{I})$ (almost no downshift) but weaker than $\mathrm{H}_{2}$ (II) (downshift by ca. $560 \mathrm{~cm}^{-1}$ ). Thus we consider $\mathrm{H}_{2}$ (III) to be located in a defect created by ion implantation, whose size is greater than the tetrahedral site but smaller than the platelet site.

Candidates for such traps are multivacancies and interstitial complexes. An electron paramagnetic resonance study on intrinsic silicon implanted with $160-\mathrm{keV} \mathrm{O}^{+}$ions, for example, confirmed the formation of divacancy and four-vacancy. ${ }^{10}$ The four-vacancy was observed at fluences between $2 \times 10^{12}$ and $2 \times 10^{14} \mathrm{O}^{+} / \mathrm{cm}^{2}$, with a maximum at around $2 \times 10^{13} \mathrm{O}^{+} / \mathrm{cm}^{2}$, while divacancy and localized

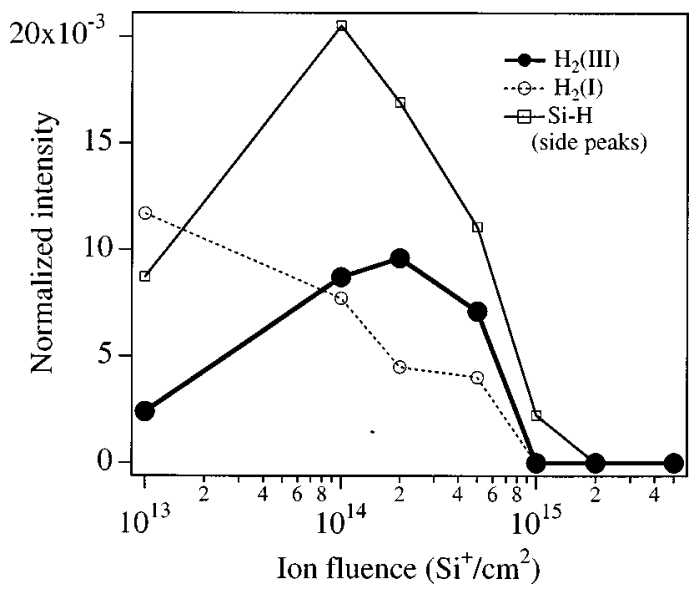

FIG. 2. Raman intensities of the $\mathrm{H}_{2}$ vibrational lines and the sum of the $\mathrm{Si}-\mathrm{H}$ side peaks in silicon after implantation with $200-\mathrm{keV} \mathrm{Si}^{+}$ions at different fluences followed by a $\mathrm{H}$ atom treatment at $250{ }^{\circ} \mathrm{C}$ for $3 \mathrm{~h}$. 


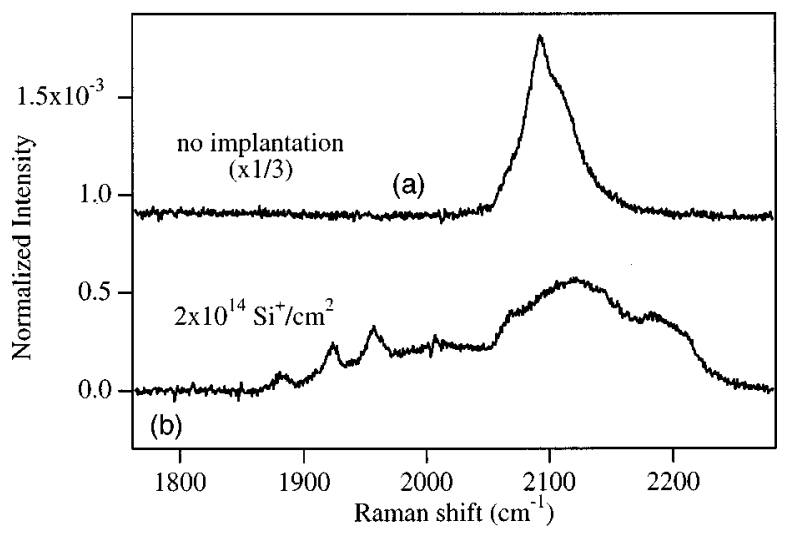

FIG. 3. Raman spectra of (a) crystalline silicon after a $\mathrm{H}$ atom treatment at $250^{\circ} \mathrm{C}$ for $3 \mathrm{~h}$, and (b) silicon after implantation with $200-\mathrm{keV} \mathrm{Si}^{+}$ions at a fluence of $2 \times 10^{14} / \mathrm{cm}^{2}$ followed by a $\mathrm{H}$ atom treatment at $250{ }^{\circ} \mathrm{C}$ for $3 \mathrm{~h}$.

amorphous regions are formed effectively at lower and higher fluences, respectively. This study suggests that multivacancies larger than the divacancy are effectively created in the ion fluence range in which $\mathrm{H}_{2}$ (III) was observed in the present study. The Raman spectra of $\mathrm{Si}-\mathrm{H}$ stretching in unimplanted silicon and silicon implanted with $2 \times 10^{14} \mathrm{Si}^{+} / \mathrm{cm}^{2}$ are compared in Fig. 3, both after the hydrogen atom treatment at $250^{\circ} \mathrm{C}$. The Si-H Raman band for implanted silicon exhibits several side peaks, three at the low-frequency side $\left(1883,1926\right.$, and $\left.1957 \mathrm{~cm}^{-1}\right)$ and at least two at the high-frequency side (2187 and $\left.2213 \mathrm{~cm}^{-1}\right)$. These side peaks are similar to those attributed to hydrogenterminated dangling bonds observed in hydrogen-implanted $\mathrm{Si}^{15}$ Some of the side peaks are also possibly originated from complexes of Si interstitials and hydrogen. ${ }^{16}$ The integrated total intensity of the above-mentioned side peaks is plotted in Fig. 2. The ion-fluence dependence of the Si-H side peaks exhibits an evident correlation with that of $\mathrm{H}_{2}(\mathrm{III})$, indicating that multivacancies and interstitial complexes play an important role in the formation of $\mathrm{H}_{2}$ (III).

Calculations have shown that $\mathrm{H}_{2}$ is stable in multivacancies with and without $\mathrm{H}$ termination. The vibrational frequency of $\mathrm{H}_{2}$ in $\mathrm{H}$-terminated divacancy $\left(\mathrm{V}_{2} \mathrm{H}_{6}\right)$ was approximately $3800 \mathrm{~cm}^{-1}$, assuming $\mathrm{H}_{2}$ is located at the Td site adjacent to the $\mathrm{Si}-\mathrm{H}$ bonds in the divacancy. ${ }^{17}$ The vibrational frequency was calculated to be approximately 4000 $\mathrm{cm}^{-1}$ for $\mathrm{H}_{2}$ in six and ten vacancies $\left(V_{6} \mathrm{H}_{12}\right.$ and $\left.V_{10} \mathrm{H}_{16}\right)$ with $\mathrm{H}$ termination, ${ }^{17,18}$ and $3780 \mathrm{~cm}^{-1}$ without $\mathrm{H}$ termination. ${ }^{18}$ These calculations agree roughly with the experimentally observed frequency of $\mathrm{H}_{2}(\mathrm{III}), 3822 \mathrm{~cm}^{-1}$, and supports its assignment as a hydrogen molecule trapped by $\mathrm{H}$-terminated multivacancies in silicon. It is noted that the width of the $\mathrm{H}_{2}$ (III) line is about $30 \mathrm{~cm}^{-1}$, being comparable to that of $\mathrm{H}_{2}(\mathrm{I})$ and much broader than that of $\mathrm{H}_{2}(\mathrm{II})$. The large width is accounted for by inhomogeneous broadening; hydrogen molecules are plausibly trapped in multivacancies with several different sizes (divacancy, four vacancy, five vacancy, etc.), or located at several metastable sites in or adjacent to the same multivacancy.

In conclusion, we have presented the existence of a $\mathrm{H}_{2}$ molecule trapped by multivacancies in ion-implanted silicon. The ion-fluence dependence and the isotope shifts confirmed the assignment. The vibrational frequency of the $\mathrm{H}_{2}$ was $3822 \mathrm{~cm}^{-1}$, between the two Raman lines observed in crystalline silicon, 4158 and $3601 \mathrm{~cm}^{-1}$. This result encourages us to further investigate hydrogen molecules existing in a defective environment in silicon that have not yet been investigated.

The authors would like to thank A. Oshiyama for theoretical calculations. They also thank S. Fujimura and J. Kikuchi for their help in setting up the hydrogen atom treatment apparatus, and H. Haneda for his SIMS measurement of the samples.
*Present address: Material Research Institute, Tohoku Univ. Katahira, Sendai, Japan.

${ }^{1}$ K. Murakami, N. Fukata, S. Sasaki, K. Ishioka, M. Kitajima, S. Fujimura, J. Kikuchi, and H. Haneda, Phys. Rev. Lett. 77, 3161 (1996)

${ }^{2}$ J. Vetterhöffer, J. Wagner, and J. Weber, Phys. Rev. Lett. 77, 5409 (1996).

${ }^{3}$ N. Fukata, S. Sasaki, K. Murakami, K. Ishioka, K. G. Nakamura, M. Kitajima, S. Fujimura, J. Kikuchi, and H. Haneda, Phys. Rev. B 56, 6642 (1997).

${ }^{4}$ A. W. R. Leitch, V. Alex, and J. Weber, Solid State Commun. 105, 215 (1997).

${ }^{5}$ R. E. Prichard, M. J. Ashwin, R. C. Newman, J. H. Tucker, E. C. Lightowlers, M. J. Binns, R. Falster, and S. A. McQuiaid, Phys. Rev. B 56, 13118 (1997); R. E. Prichard, M. J. Ashwin, J. H. Tucker, and R. C. Newman, ibid. 57, 15048 (1998).

${ }^{6}$ A. W. R. Leitch, V. Alex, and J. Weber, Phys. Rev. Lett. 81, 421 (1998).

${ }^{7}$ Y. Okamoto, M. Saito, and A. Oshiyama, Phys. Rev. B 56, R10 016 (1997).

${ }^{8}$ C. G. Van de Walle, Phys. Rev. Lett. 80, 2177 (1998).

${ }^{9}$ B. Hourahine, R. Jones, S. Öberg, R. C. Newman, P. R. Briddon, and E. Roduner, Phys. Rev. B 57, R12 666 (1998).
${ }^{10}$ K. L. Brower and W. Beezhold, J. Appl. Phys. 43, 3499 (1972).

${ }^{11}$ K. Murakami, K. Masuda, K. Gamo, and S. Namba, Jpn. J. Appl. Phys. 12, 1307 (1973).

${ }^{12}$ H. Richter, Z. P. Wang, and L. Ley, Solid State Commun. 39, 625 (1981).

${ }^{13}$ M. Kitajima, K. Ishioka, S. Tateishi, N. Fukata, K. Murakami, S. Fujimura, S. Hishita, M. Komatsu, and H. Haneda, Mater. Sci. Eng. A 130-132, 243 (1999); M. Kitajima, K. Ishioka, K. Nakanoya, S. Tateishi, T. Mori, N. Fukata, K. Murakami, and S. Hishita, Jpn. J. Appl. Phys. Part 2 38, L691 (1999).

${ }^{14}$ N. Fukata, S. Fujimura, and K. Murakami, Mater. Sci. Forum 196-201, 873 (1995).

${ }^{15}$ M. K. Weldon, V. E. Marsico, Y. J. Chabal, A. Agarwal, D. J. Eaglesham, J. Sapjeta, W. L. Brown, D. C. Jacobson, Y. Caudano, S. B. Christman, and E. E. Chaban, J. Vac. Sci. Technol. B 15, 1065 (1997).

${ }^{16}$ For example, M. Budde, B. B. Nielsen, P. Leary, J. Goss, R. Jones, P. R. Briddon, S. Oberg, and S. J. Breuer, Phys. Rev. B 57, 4397 (1998).

${ }^{17}$ T. Akiyama, Y. Okamaoo, M. Saito, and A. Oshiyama (unpublished).

${ }^{18}$ M. Nimura (private communication). 